\title{
Receiver Stimulator Unit Device
}

National Cancer Institute

\section{Source}

National Cancer Institute. Receiver Stimulator Unit Device. NCI Thesaurus. Code C50330.

As part of a cochlear implant, an implanted device designed to receive signal from the external device and then decode the signal and transmit it to the brain. 